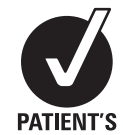

CHOICE

\title{
Coping with amyotrophic lateral sclerosis: an integrative view
}

\author{
Tamara Matuz, ${ }^{1}$ Niels Birbaumer, ${ }^{1,2}$ Martin Hautzinger, ${ }^{3}$ Andrea Kübler ${ }^{1,4}$
}

\begin{abstract}
See Editorial Commentary, p 826

${ }^{1}$ Institute of Medical Psychology and Behavioral Neurobiology, Eberhard-Karls-University Tübingen, Germany

${ }^{2}$ Istituto di Ricovero e Cura a Carattere Scientifico (IRCCS), Ospedale San Camillo, Venezia, Lido, Italy

${ }^{3}$ Department of Psychology, Eberhard-Karls-University Tübingen, Germany

${ }^{4}$ Department of Psychology I, Biological Psychology, Clinical Psychology, and Psychotherapy, University of Würzburg, Germany
\end{abstract}

\section{Correspondence to}

Dr Tamara Matuz, Eberhard-Karls-University Tübingen, Institute of Medical Psychology and Behavioral Neurobiology, Gartenstr. 29, Tübingen 72074, Germany; tamara.matuz@medizin. uni-tuebingen.de

Received 24 November 2009 Revised 25 February 2010 Accepted 26 February 2010 Published Online First 28 June 2010

\section{ABSTRACT}

Objectives To identify predictors of psychosocial adjustment to motor neurone disease.

Methods A total of 27 individuals with a confirmed diagnosis of amyotrophic lateral sclerosis (ALS) participated in the study. The ALS functional rating scale mean score indicated a high physical impairment of the sample. Months since diagnosis varied between 4 and 129 (median 36). Adjustment outcomes were severity of depressive symptoms and individual quality of life ( $\mathrm{OoL})$. Predictors included social support, cognitive appraisal, coping strategies and illness parameters.

Results Multiple regression analysis revealed that approximately $60 \%$ of the variance of depression and OoL were accounted for by social support, coping strategies and cognitive appraisal. The degree of physical impairment did not explain any variance of the adjustment outcomes. The best predictors for the severity of depressive symptoms were perceived social support and appraisal of coping potential (internal locus of control) and for individual OoL perceived social support.

Conclusions The focus on medical issues in treatment of ALS is not sufficient. A palliative approach to ALS must equally imply advice with regards to adequate coping strategies, provide the adequate amount of disease- and support-related information at any one time, and encourage patients to seek social support. Sufficient medication and psychotherapy has to be provided for those patients who show depressive symptoms or disorder.

\section{INTRODUCTION}

People with fatal diseases, such as HIV, cancer, multiple sclerosis (MS) or amyotrophic lateral sclerosis (ALS), are permanently challenged by disease progression. The impact of symptoms (in ALS and MS, particularly, the increasing physical impairment due to motor paralyses) and the lack of a curative treatment requires adaptive mechanisms. In the context of chronic illness and physical disability, the person-environment equilibrium is disrupted and for its restoration patients have to adjust their internal needs to the new external demands. This process has been referred to as the psychosocial adjustment to chronic illness and disabilities. ${ }^{1}$

There is a general agreement that despite the continuously increasing physical impairment, ALS patients adjust effectively to their illness and are often perceived as particularly positive people. These observations are supported by a number of studies investigating different adjustment variables-for example, quality of life, depression or anxiety, social support ${ }^{2}$ - as well as by a few studies exploring the neural correlates of emotional processing in $\mathrm{ALS}^{3}{ }^{4}$

Behavioural and emotional changes in motor neuron disease (MND), when present, have been shown to be partially in line with the spectrum of symptoms that characterise the behavioural variant of frontotemporal dementia (bvFTD) (eg, apathy, self-centredness, blunting of emotions). ${ }^{5}$ However, findings of imaging studies on emotional processing in ALS could not confirm a causal relation between the altered emotional responses in ALS (eg, more positive valence and decreased excitability to extreme emotional stimuli) and frontal lobe dysfunction. $^{3}$

Although there are several studies investigating different psychosocial aspects of MND/ALS, to our knowledge, no effort has been made to integrate the different determinants of psychosocial adjustment into a model of coping with ALS. To have a model of psychosocial adjustment to ALS would be of value to guide intervention and treatment when patients fail to cope with the disease leading to depression, poor quality of life (QoL) and the wish for a hastened death. ${ }^{6}$ The current study aimed at providing such a model. As we assumed that living with ALS constitutes a stressful event in life, we based our approach on a stress-coping model (eg, Lazarus and Folkman ${ }^{7}$ ) adapted to chronic diseases. ${ }^{8} 9$ According to this model, psychosocial adjustment to illness is determined by a complex interaction between four variables: illness parameters, cognitive appraisal, coping resources and coping strategies. Possible outcome measures of psychosocial adjustment are the affective state and quality of life. ${ }^{10}$

The objective of the present study was, first, to examine the use of Lazarus and Folkman's stresscoping model - that is how much of the variance in psychosocial adjustment could be explained by the model in a sample of people with ALS. Second, to determine to what extent the model could predict successful psychological adjustment to ALS.

\section{METHODS \\ Participants and procedure}

The study was approved by the Ethical Committee of the Medical Faculty, University of Tübingen. The inclusion criterion for patients was a neurologist's diagnosis of ALS. Patients with diagnosed frontotemporal dementia, alcoholism and poor knowledge of the German language were excluded. Twenty-seven ALS patients (mean age \pm SD: $55.3 \pm 11.1 ; 35-73 ; 12$ women) were included. Fifteen patients were recruited from the ALS outpatient clinic of the Department of Neurology, University of Ulm, six from the Institute of 
Medical Psychology and Behavioural Neurobiology, University of Tübingen, and six through an appeal for participation in the study in a biannual magazine published by the German Society for Muscular Diseases. All patients were visited at their homes by a clinical psychologist (TM), signed informed consent forms and were interviewed. Patients were interviewed alone except those $(\mathrm{N}=2)$ who used eye movement for communication and needed occasional communicative support by caregivers or family members in case the interviewer could not decode/ understand the message. According to the interviewer, the presence of the third person did not influence the respondent's answers. However, an eventual bias can not be totally excluded based on this assessment.

\section{Measures}

\section{Background data}

Patients' demographic data, including age, sex, marital status, level of education, living arrangement and communication abilities were obtained with a semi-structured interview.

\section{Illness parameters}

Three illness related variables were assessed: duration of illness (month since diagnosis), dependence on life-sustaining treatment (ventilation and nutrition) and physical disability (ALS Function Rating Scale ${ }^{11}$ with scores range from $0=$ complete paralysis to $40=$ normal physical functioning).

\section{Appraisal components}

Patients' primary and secondary appraisals were assessed with four face-valid items designed to measure motivational relevance, motivational congruence, problem focused and emotion focused coping potential. ${ }^{12}$ Patients rated each item on a 9-point Likert scale ( 1 referred to not at all and 9 to extremely). The larger the difference between the two items of primary appraisal 'motivational relevance' and 'motivational congruence' the more patients feel threatened by the disease.

\section{Social support}

The Berlin Social Support Scales (BSSS) ${ }^{13}$ was developed based on a multidimensional approach and includes five subscales measuring both cognitive and behavioural aspects of social support: perceived available social support, actually received social support, need for support, search for support and protective buffering. Patients rated their agreement with the statements from each of the scales on a 4-point Likert scale ranging from strongly disagree (1) to somewhat disagree (2), somewhat agree (3) and strongly agree (4).

\section{Coping strategies}

Coping strategies can be classified in four categories: problemmanagement, problem-appraisal, emotion-management and emotional-avoidance. ${ }^{14}$ Problem-management coping strategies describe active attempts to manage the situation (eg, seek for information) whereas problem-appraisal coping involves attempts to avoid a direct and active confrontation with the problem by either reappraisals of the stressfulness of the situation (eg, positive thinking) or by behavioural distraction (eg, positive acting). Emotion-management strategies can be conceptualised as efforts to gain knowledge, to understand and to express emotions engendered by the situation whereas emotional avoidance represents the active attempts to avoid emotions induced by the stressful event. ${ }^{14}{ }^{15}$ Coping strategies were assessed with the Motor Neuron Disease Coping Scale (MNDCS). ${ }^{16}$ Eighteen items were assigned to six subscales.
Patients expressed the extent in which they relied on the coping strategies in the last month using a 6-point Likert scale. We integrated the six subscales of this measure in the classification of coping strategies described above as follows: information seeking and support scales as problem management strategies; positive thinking and positive action scales as problem appraisal strategies; independence scale as emotion management coping and avoidance as emotional avoidance strategy. One score for each type of coping was obtained by generating the mean score of the grouped scales.

\section{Depressive symptomatology}

Severity of depressive symptoms was assessed with Beck's Depression Inventory. ${ }^{17}$ Values below 11 indicate no, values between 11 and 18 mild to moderate, and values above 18 clinically relevant severity of depressive symptoms.

\section{Individual quality of life}

The schedule for the Evaluation of Individual Quality of LifeDirect Weighting (SEIQoL-DW) ${ }^{18}$ allows the patients to propose five areas of their life that they consider most relevant for their QoL. They then indicate the relative importance of each area and then the degree of satisfaction with each of the areas. The resulting SEIQoL index (see results) may range from 0 (worst possible QoL) to 100 (best possible QoL).

\section{Statistical analysis}

Normal distribution of the data was tested with KolmogorovSmirnov Test. We report the median when data were not normally distributed. With continuous data, correlational analyses were performed to examine whether the dependent and independent variables varied as a function of the background data. Mann-Whitney U test and Kruskal-Wallis H-test were applied to categorical data with the same purpose. Non-parametric tests were used with regard to the small sample size and when data were not normally distributed. To compare two variables within the same participants, paired-samples t-test or $Z$ Wilcoxon signed-rank test were used. For the comparison of more than two conditions in the same participants, analysis of variance (ANOVA) or Friedman's ANOVA was computed. To evaluate the relative impact of the predictor variables on the measures of psychosocial adjustment, two multiple regression analyses were conducted separately for depression and QoL scores.

Each predictor was assessed through multiple subscales and, thus, the predictors:sample size ratio was inadequate for regression modelling (the predictors exceeded the sample size). To reduce the number of predictors, multiple linear regressions (stepwise) were first performed with the subscales of each predictor's variable separately. Those subscales that showed an adjusted (Bonferroni corrected at $\mathrm{p}<0.01$ ) significant contribution to the prediction of the dependent variable were included in the final regression. Analysis of the model assumptions (ie, independence of the residuals, absence of the outliers, multicollinearity, linearity and homoscedasticity) was performed. We used SPSS 15.0 for all statistical analyses.

\section{RESULTS}

\section{Background data and adjustment outcomes}

Background and disease related data are listed in table 1.

The group's $\mathrm{BDI}$ mean score was 11.8 (SD \pm 6.9 ; range 1-29) and five patients $(18.5 \%)$ had clinically relevant depressive symptoms. Mean score of individual OoL was 67.8 ( $\mathrm{SD} \pm 15.8$; 
Table 1 Background and disease-related data of the patient sample

\begin{tabular}{|c|c|c|c|c|c|c|c|c|c|}
\hline \multicolumn{2}{|c|}{ Education level } & \multicolumn{2}{|c|}{ Marital status } & \multicolumn{2}{|c|}{ Living environment } & \multicolumn{2}{|l|}{ Sex } & \multicolumn{2}{|c|}{ Communication* } \\
\hline Higher & 13 & Married & 21 & Own household & 25 & Male & 15 & Normal & 7 \\
\hline \multirow[t]{3}{*}{ Lower } & 14 & Single & 3 & Nursing home & 2 & Female & 12 & Impaired & 15 \\
\hline & & Widowed & 2 & & & & & With devices & 3 \\
\hline & & Divorced & 1 & & & & & & \\
\hline \multicolumn{2}{|c|}{ Onset of ALS } & \multicolumn{2}{|c|}{ Type of ALS } & \multicolumn{2}{|c|}{ Artificial ventilation } & \multicolumn{2}{|c|}{ Invasive ventilation } & PEG & \\
\hline Bulbar & 2 & Sporadic & 27 & Yes & 11 & Yes & 3 & Yes & 6 \\
\hline Spinal & 25 & Genetic & 0 & No & 18 & No & 24 & No & 21 \\
\hline
\end{tabular}

*Normal communication refers to normal speech process; impaired communication ranged from 'detectable speech disturbance' to 'communication intelligible with repeating'; with devices referring to loss of useful speech and communication exclusively possible by using assistive technology and/or eye movements.

ALS, amyotrophic lateral sclerosis; PEG, percutaneous endoscopic gastrostomy.

range 32-91). Age correlated significantly positive with QoL $(\mathrm{r}=0.49 ; \mathrm{p}<0.01$; Pearson's correlation), but not with depression $(r=0.09, p=0.7$, Pearson's correlation). Depression and QoL did not differ between sexes (depression: $U=71, p=0.3$; OoL: $\mathrm{U}=57.5, \mathrm{p}=0.1$; Mann-Whitney $\mathrm{U}$ tests) and as a function of education (depression: $U=68, p=0.2$; QoL: $U=56, p=0.09$; Mann-Whitney U tests). Married patients and patients living in partnership reported significantly higher QoL as compared to patients living without a partner $(\mathrm{U}=15.5, \mathrm{p}=0.01$; Mann-Whitney U tests). There was no difference between these groups regarding depression scores $(U=36.5, p=0.2$; Mann-Whitney U tests).

\section{Predictors of psychosocial adjustment: descriptive and comparative analysis}

Mean, median, SD and range for all predictor variables are listed in table 2. With a mean score of 17.4, ALS-Functional Rating Scale (ALS-FRS) values indicated high physical impairment of the patient sample. Correlation analysis revealed that neither severity of depressive symptoms nor QoL correlated significantly with time since diagnosis and the degree of physical impairment. To compare patients with and without life-sustaining treatment, Mann-Whitney U tests were conducted and proved non-significant. For all coefficients and probabilities see table 3 .

Scores of received and perceived social support indicated a high availability of social support in this sample (table 2). Median of primary appraisals evidenced that patients rated their illness situation as being not threatening (table 2). Significant mean differences were found between the appraisals of problemfocused coping potential and emotion-focused coping potential $\left(t_{(26)}=-4.5 ; \mathrm{p}<0.001 ;\right.$ paired sample $\mathrm{t}$ test $)$, which meant that patients dealt better with the situation emotionally than by active attempts to change it. However, patients rated both coping potentials as being high indicating that they experienced a high propensity to cope well with their disease.

Friedman's ANOVA revealed significant differences between the frequencies of using coping strategies $\left(\chi_{(3)}^{2}=45.2, p<0.0001\right.$ Friedman's ANOVA). Wilcoxon signed-rank tests were used for pair-wise comparisons (Bonferroni corrected) and revealed that emotional avoidance was less frequently used then all the other coping strategies (all $\mathrm{p}<0.001$ ). Moreover, patients relied less frequently on problem-management and problem-appraisal strategies than on emotion-management strategies $(Z=-2.7$, $\mathrm{p}<0.008 ; Z=-2.6, \mathrm{p}<0.008$; Wilcoxon signed rank tests).

\section{Data reduction and selection of predictors}

Regression analyses were conducted separately for depression and GoL with the subscales (aspects) of each stress-coping model predictor to (1) investigate the relation between the aspects of the predictor variables and adjustment outcome, and (2) to reduce and select predictors for the final regression analysis. The coefficients are presented in table 4.

\section{IIIness characteristics}

Regression analysis revealed that neither severity of depressive symptoms nor QoL could be significantly predicted by the time since diagnosis and the degree of physical impairment (table 4); thus, these parameters were not included in the final regression.

\section{Social support}

The subscales on received social support and perceived social support of BSSS were included in the regression analysis because these subscales are geared towards assessment of social support as a coping resource. The other scales of the instrument were related to coping behaviour and, thus, were not included in the final regression to avoid overlap with the coping strategy

Table 2 Predictor variables: mean (M), median (Mdn), SD, range, maximum possible values and group differences depending on the time since diagnosis

\begin{tabular}{|c|c|c|c|c|c|}
\hline \multicolumn{2}{|c|}{ Predictors of psychosocial adjustment } & \multirow{2}{*}{$\begin{array}{l}\text { M/Mdn } \\
17.4\end{array}$} & \multirow{2}{*}{$\begin{array}{l}\text { SD } \\
9.8\end{array}$} & \multirow{2}{*}{$\begin{array}{l}\text { Range } \\
0-36\end{array}$} & \multirow{2}{*}{$\begin{array}{l}\text { Maximum possible } \\
40\end{array}$} \\
\hline Illness charac teristics & Physical impairment & & & & \\
\hline & Time since diagnosis & 36 & & $4-129$ & - \\
\hline \multirow[t]{4}{*}{ Social support } & Perceived & 4 & & $2.1-4$ & 4 \\
\hline & Need for support & 2.9 & 0.7 & $1.2-4$ & 4 \\
\hline & Search for support & 2.8 & 0.6 & $1.2-4$ & 4 \\
\hline & Protective buffering & 2.2 & 0.6 & $1.1-3.5$ & 4 \\
\hline \multirow[t]{4}{*}{ Coping strategies } & Problem management & 4.1 & & $2.1-5$ & 5 \\
\hline & Problem appraisal & 4.2 & & $2-5$ & 5 \\
\hline & Emotion management & 4.5 & & $3.5-5$ & 5 \\
\hline & Emotional avoidance & 2.3 & 0.8 & $0.6-4$ & 5 \\
\hline
\end{tabular}


Table 3 Adjustment outcomes and illness parameters

\begin{tabular}{lllllll}
\hline & & Illness parameters & & & \\
\cline { 3 - 7 } & & Physical impairment & Time since diagnosis & Artificial nutrition & Artificial ventilation & Invasive ventilation \\
\hline Adjustment outcomes & Depression & $r=-0.2 p=0.3$ & $\tau=0.01 p=0.9$ & $U=61 p=0.9$ & $U=73 p=0.4$ & $U=28.5 p=0.5$ \\
& CoL & $r=0.01 p=0.9$ & $\tau=0.14 p=0.2$ & $U=57 p=0.7$ & $U=82 p=0.7$ & $U=18 p=0.1$ \\
\hline
\end{tabular}

$r$, Pearson's correlation; $\tau$, Kendall's correlation; QoL, quality of life; U, Mann-Whitney U-Test.

subscales. Results showed that higher perceived social support could significantly predict lower depression and higher QoL (table 4). Thus, the scale on perceived social support was selected as a predictor for the final regression.

\section{Coping strategies}

Higher scores on the scale independence significantly predicted lower severity of depressive symptoms (table 4). Higher QoL scores were significantly predicted by problem-management and avoidant-type of strategies. The emotion-management strategy (independence) for depression and the problem-management and avoidant type of strategies, for QoL, were included in the final regression analyses.

\section{Cognitive appraisal}

The results of the regression analysis showed that higher appraisals of both emotion and problem focused coping potential, which can be interpreted as higher internal locus of control, predicted lower severity of depressive symptoms (table 4). The variance of the QoL scores could not be significantly accounted for by any of the appraisals scales.

\section{Regression analysis}

A multiple regression analysis was performed on each of the two dependent variables. For depression, the block of predictors included perceived social support, appraisal of coping potential and emotion management coping (independence scale of the MNDCS). For QoL, we included perceived social support, problem-management coping strategies (information seeking and support) and avoidance. Since no hierarchical assumptions about these predictors were made, all of them were entered in one step using the method Enter. A summary of the regression analyses is presented in table 5. Assumptions (see methods) were tested and met. The regression analysis showed that $61 \%$ of the variance of severity of depressive symptoms was accounted for by all the predictor variables taken together. The $\beta$ values (table 5) indicated the independent contribution of each predictor to the total explained variance. Hence, perceived social support and appraisal of coping potential individually accounted for a significant percentage in the variance in severity of depressive symptoms. Higher perceived social support $\left(t_{(27)}=-2.28 ; p<0.05\right)$ and more confidence in their own coping potential $\left(\mathrm{t}_{(27)}=-2.49 ; \mathrm{p}<0.05\right)$ predicted lower depressed mood. None of the coping strategies predicted the severity of depressive symptoms.

Selected predictors explained $56 \%$ of the variance in $\mathrm{QoL}$ $\left(F_{(3,23)}=9.87 ; \mathrm{p}<0.001\right) . \beta$ Values indicated that higher perceived social support $\left(\mathrm{t}_{(27)}=3.54 ; \mathrm{p}<0.01\right)$ predicted higher $\mathrm{OoL}$. Patients who searched more frequently for information and support $\left(\mathrm{t}_{(27)}=2.5 ; \mathrm{p}<0.05\right)$ and showed more avoidant behaviour $\left(\mathrm{t}_{(27)}=3.11 ; \mathrm{p}<0.05\right)$ also reported higher $\mathrm{QoL}$. However, these relation did not reach significance at the corrected $p$ value. The results of the final regression analysis are depicted in figure 1.

\section{DISCUSSION}

Perceived social support, appraisal of coping potential and independence all together explained $61 \%$ of the variance of

Table 4 Summary of regression analyses on the two adjustment variables with the subscales of each stress-coping predictors separately

\begin{tabular}{|c|c|c|c|c|c|c|c|}
\hline \multirow[b]{2}{*}{ Predictors } & \multirow[b]{2}{*}{ Dependent variables } & \multicolumn{3}{|c|}{ Depression } & \multicolumn{3}{|c|}{ Quality of life } \\
\hline & & $\overline{\mathbf{B}}$ & SE B & $\beta$ & $\bar{B}$ & SE B & $\beta$ \\
\hline \multicolumn{8}{|c|}{ Social support } \\
\hline & Perceived & -5.5 & 3.03 & $-0.54^{* *}$ & 14.3 & 4.4 & $0.44^{* *}$ \\
\hline & Received & -2.9 & 2 & -0.24 & 4.9 & 6.7 & 0.23 \\
\hline & & \multicolumn{3}{|c|}{$\begin{array}{l}\mathrm{R}^{2}=0.33, \Delta \mathrm{R}^{2}=0.28 \\
\mathrm{~F}_{(2,24)}=6.03, \mathrm{p}<0.01\end{array}$} & \multicolumn{3}{|c|}{$\begin{array}{l}\mathrm{R}^{2}=0.37, \Delta \mathrm{R}^{2}=0.32 \\
\mathrm{~F}_{(2,24)}=7.2, \mathrm{p}<0.01\end{array}$} \\
\hline \multicolumn{8}{|c|}{ Cognitive appraisal } \\
\hline & Primary appraisal & -0.24 & 0.42 & -0.11 & -0.2 & 1.1 & -0.004 \\
\hline & Appraisal of coping potential & -2.4 & 0.77 & $-0.56^{* *}$ & 1.33 & 2.1 & 0.15 \\
\hline & & \multicolumn{3}{|c|}{$\begin{array}{l}\mathrm{R}^{2}=0.31, \Delta \mathrm{R}^{2}=0.26 \\
\mathrm{~F}_{(2,24)}=5.6, \mathrm{p}<0.01\end{array}$} & \multirow{2}{*}{\multicolumn{3}{|c|}{$\begin{array}{l}\mathrm{R}^{2}=0.02, \Delta \mathrm{R}^{2}=0.06 \\
\mathrm{~F}_{(2,24)}=0.02, \mathrm{p}=0.8\end{array}$}} \\
\hline \multicolumn{7}{|c|}{ Coping strategies } & \\
\hline & Problem management & -1.6 & 1.6 & -0.22 & 9.57 & 3.9 & $0.44^{* *}$ \\
\hline & Problem appraisal & -2.1 & 1.3 & -0.32 & 2.67 & 3.1 & 0.15 \\
\hline & Emotion management & -5.1 & 2.7 & $-0.53^{* *}$ & -8.74 & 6.4 & -0.26 \\
\hline & Emotional avoidance & -0.62 & 1.4 & -0.11 & 7.76 & 3.4 & $.39 *$ \\
\hline & & \multicolumn{3}{|c|}{$\begin{array}{l}\mathrm{R}^{2}=0.40, \Delta \mathrm{R}^{2}=0.29 \\
\mathrm{~F}_{(4,22)}=9.8, p<0.01\end{array}$} & \multirow{2}{*}{\multicolumn{3}{|c|}{$\begin{array}{l}R^{2}=0.38, \Delta R^{2}=0.28 \\
F_{(4,22)}=3.48, p<0.05\end{array}$}} \\
\hline \multicolumn{7}{|c|}{ Illness parameters } & \\
\hline & Physical impairment & -0.12 & 0.15 & -0.17 & 0.08 & 034 & 0.05 \\
\hline & Time since diagnosis & 0.02 & 0.05 & 0.07 & 0.06 & 0.11 & 0.11 \\
\hline & & \multicolumn{3}{|c|}{$\begin{array}{l}\mathrm{R}^{2}=0.04, \Delta \mathrm{R}^{2}=0.03 \\
\mathrm{~F}_{(1,24)}=0.04, p=0.6\end{array}$} & \multicolumn{3}{|c|}{$\begin{array}{l}\mathrm{R}^{2}=0.01, \Delta \mathrm{R}^{2}=0.07 \\
\mathrm{~F}_{(1,24)}=0.1, \mathrm{p}=0.9\end{array}$} \\
\hline
\end{tabular}


Table 5 Summary of regression analyses of stress-coping variables predicting psychological adjustment to amyotrophic lateral sclerosis

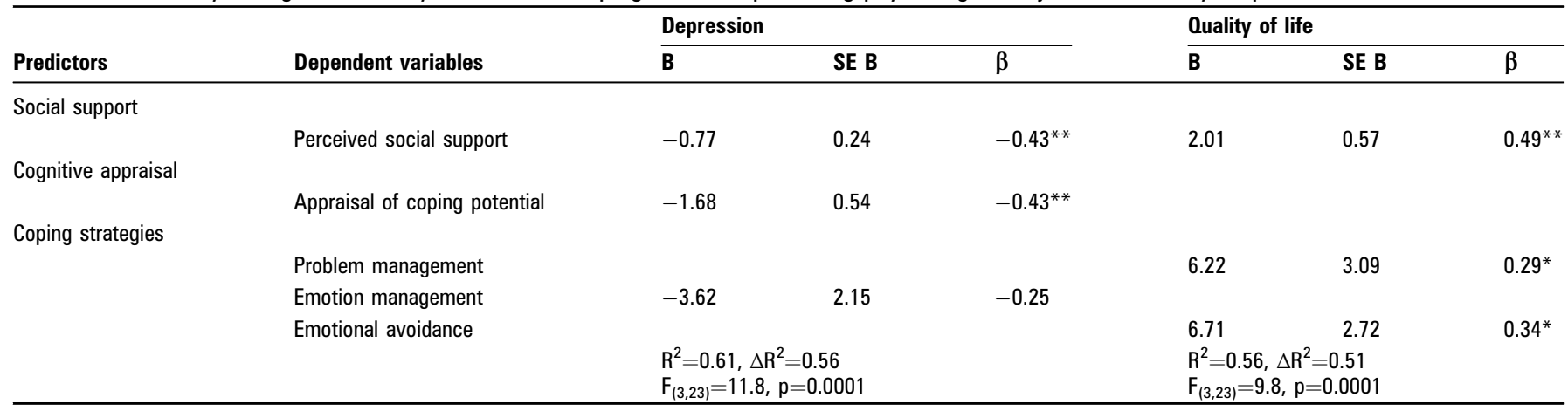

${ }^{*} \mathrm{p}<0.05 ;{ }^{* *} \mathrm{p}<0.01$.

$\Delta R^{2}, R^{2}$ change; $\beta$, standardised betas; $B$, b-values; $S E B$, SE of the $b$ values.

severity of depressive symptoms. Perceived social support, seeking for support, and information and avoidance accounted for $56 \%$ of the variance in QoL. The degree of physical impairment and the time since diagnosis did not explain any variance in the adjustment outcomes. This result is of utmost importance as it corroborates that more severe functional impairment does not inevitably lead to poorer QoL and depressive symptoms.

Earlier studies on psychosocial aspects in ALS report similar outcomes but mostly as a result of correlative analyses. ${ }^{19-22}$ In contrast, our study revealed the predictive power of social support, coping appraisal and coping strategies. Only a few studies aimed at predicting psychological outcomes in ALS. The outcomes referred to psychological distress (depressive symptoms and anxiety), self-esteem ${ }^{23} 24$ and hopelessness. ${ }^{25}$ We are aware of only one study trying to predict the individual $\mathrm{QoL}$ among ALS patients. ${ }^{26}$ Results of these studies are difficult to compare since they investigated different sets of predictors, such as religion, demographics, self-perceived quality of social support, present marital intimacy, which were not selected on the basis of a model to cope with disease. However, the quality

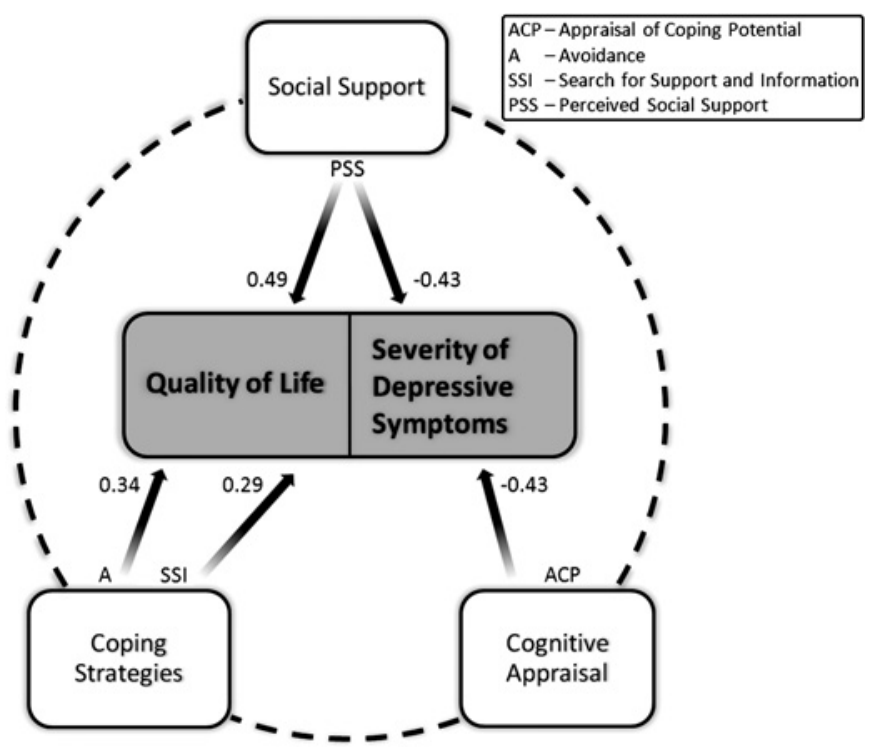

Figure 1 The modified model of psychosocial adjustment to amyotrophic lateral sclerosis on the basis of multiple regression analyses. The psychosocial predictor variables accounted together for a considerable amount of variance in both outcomes of psychosocial adjustment (depression and quality of life). This is illustrated by the dashed line. Variables that displayed independent predictive power are denoted by the arrows. of social support was evident in predicting both QoL and depressive mood, suggesting that received quality of care and the extent of how patients evaluate their social network as being supportive are of utmost importance for patients' affective state and QoL. ${ }^{23} 26{ }^{27}$ Our findings serve to further qualify this statement by demonstrating that perceived social support was the strongest predictor of QoL and severity of depressive symptoms. These results are in line with the stress and coping theory, which was originally proposed by Lazarus \& Folkman ${ }^{7}$ and has been repeatedly adapted for different chronic illnesses. ${ }^{8} 9{ }^{28}$ These models posit that appraisal determines the level of stress experienced. Higher perceived social support is presumed to interact with one's appraisal of the stressful situation to reduce the effects of stress and promote well-being. The loss of or difficulty in communication and mobility, which may lead to limited social interaction, are examples of the impairment imposed by ALS/MND that could decrease perceived social support. Therefore, comprehensive care of patients with ALS/MND should include measures that focus on maintenance of social network by providing novel communication devices and proper assistive technology. This would allow the patients to preserve their active role in the family and society.

We further found that appraisal of emotion- and problem -focused coping potential contributed significantly to the prediction of the severity of depressive symptoms in ALS, illustrating that higher internal locus of control predicts lower severity of depressive symptoms. Cognitive appraisals might have weaker effect on QoL because they rather influence emotion and subsequent coping behaviour. ${ }^{12} \mathrm{QoL}$ is a more complex psychological construct that cannot be reduced to emotional dimensions alone. QoL was better predicted by coping strategies. Searching for information and support as well as avoidance were relevant predictors of GoL such that more support and information seeking behaviour and more emotional avoidance predicted higher QoL. These results suggest that asking for instrumental and emotional support (eg, emotional intimacy and comfort; maintaining mobility with walking sticks/wheelchair) and receiving more information about the illness condition lead to higher QoL.

Although it is commonly assumed that 'confrontation is good and avoidance is bad', ${ }^{29} 30$ several studies indicated that the effects of approach and avoidance coping may vary as a function of the type of problem ${ }^{31}$ or of the stages of the illness. ${ }^{32}$ Studies of coping among cancer and HIV positive patients showed that denial (avoidant type of coping) may reduce negative emotional reactions in the early stages but may have maladaptive consequences if it leads to the postponement of medical care. ${ }^{32}$ The degenerative course of ALS exposes patients to short and 
long-term stressors, which require different strategies depending on the specific stressful aspect of the illness with which they are coping at any one time. Our results suggest that the combination of confronting and avoiding coping strategies might be beneficial for ALS patients, because more frequent search of information and for support may help them to initiate actions that are necessary to ensure optimal future care (eg, clarify support by the health insurance). On the other hand, emotional avoidance through minimising the importance of the diagnosis or directing attention away from the negative information and consequences that may occur successively as their illness progresses may prevent patients from psychological distress and despair. However, as the disease progresses, avoidance is no longer an adaptive strategy as it prevents patients from taking appropriate measures to cope further with the illness.

Among the sociodemographic variables, age and marital status showed a significant relation with QoL. Older and married ALS patients reported higher individual QoL than younger patients and patients living alone. These results are consistent with findings from studies of other diseases that lead to severe physical impairment (eg, multiple sclerosis, spinal cord injury, cancer $^{33} 34$ and studies on healthy subjects. ${ }^{35}$ During their lifespan, older people most likely get more experienced in handling stressful situations and, thus, develop a higher resistance to psychological stress ${ }^{36}$ leading to higher scores in QoL as compared to younger people. ${ }^{37}$

The main limitations of the present study are the small sample size and the high collinearity between some predictor variables, which did not allow us to include all the subscales in the regression analysis. Nevertheless, we succeeded in defining predictors of psychosocial adjustment (see figure 1). Taking into consideration at least one dimension of each stress coping predictor from Lazarus and Folkman's model, we could show that not illness-related characteristics but the psychosocial variables, first and foremost social support, best predicted psychosocial adaptation to ALS.

In summary, the present study contributes to a better understanding of psychosocial adjustment to ALS as it elucidates predictive relation between the model variables: illnessrelated parameters, coping strategies, cognitive appraisals and social support and the outcome variables depression and GoL. However, more theory driven and longitudinal research is necessary to clarify which factors have the most powerful influence on psychosocial adjustment. Such an approach would allow us to suggest and test interventions for those patients who poorly cope with the disease.

Acknowledgements We thank patients and caregivers for their readiness to participate in the study. We are grateful to Professors Christoph Braun and Boris Kotchoubey for their support in statistical analysis of the data and to Adrian Furdea for designing the figures.

Funding This study was supported by the Deutsche Forschungsgemeinschaft (DFG) through the Research Training Group 'Bioethics' at the Interdepartmental Centre for Ethics in the Sciences and Humanities (Interfakultären Zentrum für Ethik in den Wissenschaften, IZEW) of the University of Tübingen. Dr Matuz received research support in form of a PhD scholarship for 3 years.

Competing interests None

Ethics approval This study was conducted with the approval of the Ethik-Kommission der Medizinischen Fakultät und am Universitätsklinikum Tübingen

Provenance and peer review Not commissioned; externally peer reviewed.

\section{REFERENCES}

1. Livneh H, Antonak RF. Psychosocial adaptation to chronic illness and disability. Gaithersburg, MD: Aspen, 1997
2. McLeod JE, Clarke DM. A review of psychosocial aspects of motor neurone disease. J Neurol Sci 2007;258:4-10.

3. Lule D, Kurt A, Jurgens $R$, et al. Emotional responding in amyotrophic lateral sclerosis. J Neurol 2005:252:1517-24.

4. Lule D, Diekmann V, Anders S, et al. Brain responses to emotional stimuli in patients with amyotrophic lateral sclerosis (ALS). J Neurol 2007;254:519-27.

5. Lillo P, Hodges JR. Frontotemporal dementia and motor neurone disease: overlapping clinic-pathological disorders. J Clin Neurosci 2009;16:1131-5.

6. Kurt A, Nijboer F, Matuz $\mathrm{T}$, et al. Depression and anxiety in individuals with amyotrophic lateral sclerosis: epidemiology and management. CNS Drugs 2007;21:279-91.

7. Lazarus RS, Folkman S. Stress, appraisal and coping. New York: Springer, 1984.

8. Pakenham KI. Adjustment to multiple sclerosis: application of a stress and coping model. Health Psychol 1999;18:383-92

9. Pakenham KI, Dadds MR, Terry DJ. Relationships between adjustment to HIV and both social support and coping. J Consult Clin Psychol 1994;62:1194-203.

10. Anderson NB. Encyclopedia of health and behavior. California: Sage publications, 2004.

11. Cedarbaum JM, Stambler N, Malta E, et al. The ALSFRS-R: a revised ALS functional rating scale that incorporates assessments of respiratory function. BDNF ALS Study Group (Phase III). J Neurol Sci 1999;169:13-21.

12. Smith CA, Lazarus RS. Appraisal components, core relational themes and the emotions. Cogn Emot 1993; 7:233-69.

13. Schulz U, Schwarzer R. Soziale Unterstützung bei der Krankheitsbewältigung. Die Berliner Social Support Skalen (BSSS). Diagnostica 2003:49:73-82.

14. Terry DJ, Hynes GJ. Adjustment to a low-control situation: reexamining the role of coping responses. J Pers Soc Psychol 1998:74:1078-92.

15. Osowiecki DM, Compas BE. A prospective study of coping, percieved control and psychological adaption to breast cancer. Cognit Ther Res 1999;23:169-80.

16. Lee JN, Rigby SA, Burchardt F, et al. Quality of life issues in motor neurone disease: the development and validation of a coping strategies questionnaire, the MND Coping Scale. J Neurol Sci 2001;191:79-85.

17. Hautzinger $\mathbf{M b} \mathbf{M}$, Worrall H, Keller F. Das Beck Depressions-Inventar (BDI). 2 Auflag edn. Bern: Huber, 1995.

18. Hickey AM, Bury G, O'Boyle CA, et al. A new short form individual quality of life measure (SEIQOL-DW): application in a cohort of individuals with HIV/AIDS. BMJ 1996;313:29-33

19. Kübler A, Winter S, Ludolph AC, et al. Severity of depressive symptoms and quality of life in patients with amyotrophic lateral sclerosis. Neurorehabil Neural Repair 2005; 19:182-93

20. Hammer EM, Hacker S, Hautzinger M, et al. Validity of the ALS-DepressionInventory (ADI-12)- A new screening instrument for depressive disorders in patients with amyotrophic lateral sclerosis. J Affect Disord 2008:109:213-19.

21. Rabkin JG, Albert SM, Del Bene ML, et al. Prevalence of depressive disorders and change over time in late-stage ALS. Neurology 2005;65:62-7.

22. Simmons $\mathbf{Z}$, Bremer BA, Robbins RA, et al. Quality of life in ALS depends on factors other than strength and physical function. Neurology 2000;55:388-92.

23. Goldstein LH, Atkins L, Landau S, et al. Longitudinal predictors of psychological distress and self-esteem in people with ALS. Neurology 2006;67:1652-8

24. Hogg KE, Goldstein LH, Leigh PN. The psychological impact of motor neurone disease. Psychol Med 1994:24:625-32.

25. Plahuta JM, McCulloch BJ, Kasarskis EJ, et al. Amyotrophic lateral sclerosis and hopelessness: psychosocial factors. Soc Sci Med 2002:55:2131-40.

26. Chio A, Gauthier A, Montuschi A, et al. A cross sectional study on determinants of quality of life in ALS. J Neurol Neurosurg Psychiatry 2004:75:1597-601.

27. Hecht M, Hillemacher T, Grasel E, et al. Subjective experience and coping in ALS Amyotroph Lateral Scler Other Motor Neuron Disord 2002;3:225-31.

28. Stanton AL, Snider PR. Coping with a breast cancer diagnosis: a prospective study. Health Psychol 1993;12:16-23.

29. Roth S, Cohen LJ. Approach, avoidance, and coping with stress. Am Psychol 1986;41:813-19.

30. Holahan CJ, Moos RH. Life stressors, personal and social resources, and depression: a 4-year structural model. J Abnorm Psychol 1991:100:31-8.

31. Stanton AL, Danoff-Burg S, Cameron CL, et al. Coping through emotional approach: problems of conceptualization and confounding. J Pers Soc Psychol 1994:66:350-62.

32. Aldwin CM, Revenson TA. Does coping help? A reexamination of the relation between coping and mental health. J Pers Soc Psychol 1987;53:337-48.

33. Waldron D, O'Boyle CA, Kearney M, et al. Quality-of-life measurement in advanced cancer: assessing the individual. J Clin Oncol 1999;17:3603-11.

34. Hammell KW. Exploring quality of life following high spinal cord injury: a review and critique. Spinal Cord 2004;42:491-502.

35. Browne JP, O'Boyle CA, McGee HM, et al. Individual quality of life in the healthy elderly. Qual Life Res 1994;3:235-44.

36. Kunzmann U. Wisdom-Adult development and emotional-motivational dynamics In: Fernández-Ballesteros R, ed. Geropsychology. Göttingen: Hogrefe, 2007:224-38.

37. Lulé D, Häcker S, Ludolph A, et al. Depression und Lebensqualität bei Patienten mit amyotropher Lateralsklerose. Dtsch Arzteb/ 2008;105:397-403. 